\title{
Local Ocular Immunomodulation Resulting from Electrotransfer of Plasmid Encoding Soluble TNF Receptors in the Ciliary Muscle
}

\author{
Laura Kowalczuk, ${ }^{1}$ Elodie Touchard, ${ }^{1}$ Serge Camelo, ${ }^{1}$ Marie-Christine Naud, ${ }^{1}$ \\ Beatriz Castaneda, ${ }^{1}$ Nadege Brunel, ${ }^{2}$ Bernadette Besson-Lescure, ${ }^{2}$ \\ Brigitte Thillaye-Goldenberg, ${ }^{1}$ Pascal Bigey, ${ }^{3,4}$ David BenEzra, ${ }^{1,5,6}$ \\ Yvonne de Kozak, ${ }^{1}$ and Francine Behar-Coben ${ }^{1,7}$
}

Purpose. Plasmid electrotransfer in the ciliary muscle allows the sustained release of therapeutic proteins within the eye. The aim of this study was to evaluate whether the ocular production of TNF- $\alpha$ soluble receptor, using this nonviral gene therapy method, could have a beneficial local effect in a model of experimental autoimmune uveoretinitis (EAU).

METHODs. Injection of a plasmid encoding a TNF- $\alpha$ p 55 receptor $(30 \mu \mathrm{g})$ in the ciliary muscle, combined with electrotransfer $(200 \mathrm{~V} / \mathrm{cm})$, was carried out in Lewis rat eyes 4 days before the induction of EAU by S-antigen. Control eyes received naked plasmid electrotransfer or simple injection of the therapeutic plasmid. The disease was evaluated clinically and histologically. Cytokines and chemokines were analyzed in the ocular media by multiplex assay performed 15 and 21 days after immunization.

Results. Ocular TNF- $\alpha$ blockade, resulting from the local secretion of soluble receptors, was associated with delayed and significantly less severe uveitis, together with a reduction of the retinal damages. Compared with the controls, treated eyes showed significantly lower levels of IL-1 $\beta$ and MCP1, higher levels of IL-13 and IL- 4 , and reduced NOS-2 expression in infiltrating cells. Treatment did not influence TNF- $\alpha$ levels in inguinal lymph nodes.

Concuusions. Taken together, these results indicate that local immunomodulation was achieved and that no systemic adverse effects of TNF- $\alpha$ blockade observed after systemic injection of

From the ${ }^{1}$ INSERM UMRS 872 Team17, Centre de Recherches des Cordeliers, Université Pierre et Marie Curie, Université Paris Descartes, Paris, France; ${ }^{2}$ INSERM IFR65/U515, Saint Antoine Hospital, Paris, France; ${ }^{3}$ INSERM U640, CNRS UMR8151, Faculté de Pharmacie, Chemical and Genetic Pharmacology Laboratory; Université Paris-Descartes, Paris, France; ${ }^{4}$ Ecole Nationale Supérieure de Chimie de Paris, Paris, France; ${ }^{5}$ The BenEzra Eye Institute, Assouta-Rishon, Israel; the ${ }^{6}$ Department of Ophthalmology, Hadassah University Hospital, Jerusalem, Israel; and the ${ }^{7}$ Department of Ophthalmology, Hôtel-Dieu Hospital, Paris, France.

Supported by ANR Maturation and Grant LSGH-CT-2005-512036 from EVI-GenoRet.

Submitted for publication October 17, 2008; revised November 24, 2008; accepted February 24, 2009.

Disclosure: L. Kowalczuk, None; E. Touchard, None; S. Camelo, None; M.-C. Naud, None; B. Castaneda, None; N. Brunel, None; B. Besson-Lescure, None; B. Thillaye-Goldenberg, None; P. Bigey, None; D. BenEzra, None; Y. de Kozak, None; F. Behar-Cohen, None

The publication costs of this article were defrayed in part by page charge payment. This article must therefore be marked "advertisement" in accordance with 18 U.S.C. $\$ 1734$ solely to indicate this fact.

Corresponding author: Francine Behar-Cohen, Centre de Recherches des Cordeliers, UMRS 872, Team 17, Université Pierre et Marie Curie, Université Paris Descartes, F-75006 Paris, France;

behar@idf.inserm.fr.
TNF- $\alpha$ inhibitors should be expected. (Invest Ophthalmol Vis Sci. 2009;50:1761-1768) DOI:10.1167/iovs.08-3027

Tntermediate uveitis and posterior segment intraocular in1 flammatory disorders are sight-threatening eye diseases. Clinical features include inflammation of the choroid and retina with cellular infiltration and macular edema. ${ }^{1}$ Recently, polymerase chain reaction (PCR), flow cytometry, and cytokine pharmacokinetics have provided some insight into the disease mechanisms. ${ }^{2}$ Increased levels of interferon gamma (IFN- $\gamma$ ) and tumor necrosis factor-alpha (TNF- $\alpha$ ) have been found in the ocular fluids of some patients with various types of uveitis. $^{3-6}$

TNF- $\alpha$, released by macrophages and T cells during inflammation, has two distinct high-affinity receptors, p55kDa (TNFR-I) and p75kDa (TNFR-II). These receptors are expressed on the surfaces of most cells. TNF- $\alpha$ influences the secretion of interleukin-1 (IL-1), IL-6, IL-12, and IFN- $\gamma$, chemokines MCP-1/ CCL2 (chemoattractant-1) and MIP-2 (macrophage inflammatory protein-2), and adhesion molecules ELAM-1 and ICAM-1 and the production of nitric oxide (NO). Thus, TNF- $\alpha$ orchestrates inflammatory responses by stimulating the recruitment of leukocytes, activating macrophages, and driving Th- 1 cell responses within tissue. ${ }^{7}$ A key role for TNF- $\alpha$ in disease pathogenesis has been recognized in rheumatoid arthritis ${ }^{8}$ and in inflammatory bowel disease. ${ }^{9}$ Therefore, anti-TNF- $\alpha$ antibodies (infliximab, adalimumab) and a TNF- $\alpha$ p 75 receptor fusion protein (etanercept) have received United States Food and Drug Administration approval for the treatment of these diseases.

TNF- $\alpha$, produced by infiltrating cells and ocular resident cells, retinal Müller glia, retinal pigmented epithelium (RPE), and microglia, plays a key role in intraocular inflammation. In experimental autoimmune uveitis (EAU), increased ocular levels of TNF- $\alpha$ facilitate the ongoing T-cell effector responses and macrophage activation responsible for the production of proinflammatory cytokines and subsequent tissue damage. ${ }^{10}$ In EAU, systemic administration of a soluble TNF- $\alpha$ p 55 receptor reduced structural damage to the retina though the levels of inflammatory cell infiltrates remained high. ${ }^{11,12}$ Based on these findings, the therapeutic potential of systemic administration of infliximab and etanercept has been evaluated in uveitis associated with Behçet disease, juvenile arthritis, and HLAB27-associated uveitis. ${ }^{2}$ However, it has been realized that repeated systemic injections are required to maintain the initially gained visual benefits. ${ }^{13,14}$ Moreover, the secondary complications and risks associated with the systemic administration of anti-TNF- $\alpha$ agents have hampered their possible wider use in ocular inflammatory diseases. ${ }^{2,14-16}$

Because the eye is a small and relatively isolated organ, ocular gene therapy may be a potentially beneficial biotherapeutic alternative. To this aim, we have recently developed a 
novel nonviral gene therapy technology based on plasmid electrotransfer (ET) to the ciliary muscle. With this technique, high levels of soluble chimeric p 55 receptor to TNF- $\alpha$ (hTNFRIs/mIgG1) were produced in the eye. Preliminary observations demonstrated that drastic inhibition of clinical and histologic inflammation scores in rats with endotoxin-induced uveitis (EIU) could be achieved. ${ }^{17}$

In the present study, we evaluated the potential for the treatment of intraocular inflammatory and pathologic processes in the rat eye model of EAU by plasmid ET to the ciliary muscle.

\section{Materials ANd Methods}

\section{Induction and Clinical Assessment of EAU}

Male Lewis rats (6-8 weeks of age) were purchased from Janvier (Le Gesnet St. Isles, France) and roomed in our facility for 1 week before the initiation of each experiment. Animal treatment adhered to the ARVO Statement for the Use of Animals in Ophthalmic and Vision Research. Three separate sets of experiments were carried out on a total of 35 rats.

EAU was induced in rats by subcutaneous injection in the footpads of $0.2 \mathrm{~mL}$ complete Freund's adjuvant (CFA; Difco, Detroit, MI) containing $80 \mu \mathrm{g}$ S-antigen (S-Ag) supplemented with $500 \mu \mathrm{g}$ Mycobacterium tuberculosis $\mathrm{H} 37 \mathrm{Ra}$ (Difco). The S-Ag was purified from bovine retinas and emulsified (1:1) in CFA before use, as previously reported. ${ }^{18}$ Clinical disease was initially detected at the slit lamp around day 12 after immunization, reached a peak around day 15, and receded slowly thereafter.

Clinical scores of EAU were recorded by slit lamp microscopy from day 12 after immunization to the end of the experiments (to day 15 or to day 21 , during disease convalescence). The severity of uveitis was assessed in a masked manner based on the following scale: grade 0 , no signs of inflammation and normal iris dilatation after instillation of mydriaticum; grade 1, mild impairment of pupillary dilatation (up to one quadrant of posterior synechiae); grade 2, moderate impairment of pupillary dilatation (one to two quadrants of posterior synechiae); grade 3, posterior synechiae of more than two and up to four quadrants; grade 4, total seclusion of the pupil accompanied by severe iris hyperemia; grade 5, similar to grade 4 , but the aqueous humor demonstrates the presence of large amounts of fibrin; grade 6 , similar to grade 5 , but hypopion is demonstrated in the anterior chamber.

\section{Plasmids}

The pVAX2 plasmid was used as a backbone to construct the plasmid pVAX2-hTNR-Is/mIgG1, as previously described. ${ }^{19}$ This therapeutic plasmid encodes a fusion protein of human soluble TNF- $\alpha$ p 55 receptor linked to the Fc fragment of mouse immunoglobulin G1 (hTNFRIs/mIgG1). Plasmids were amplified in Escherichia coli DH5- $\alpha$ and were purified (Endofree Plasmid Giga Kit; Qiagen, Courtaboeuf, France).

Because of its excellent ability to block TNF- $\alpha$ in EIU, chimeric soluble receptor (hTNFR-Is/mIgG1) expression was used to evaluate the therapeutic efficiency of the anti-TNF- $\alpha$ strategy in EAU. To assess the intraocular production of TNF- $\alpha$ receptors, appropriate plasmid injection into the ciliary muscle combined with ET was carried out, as previously described. ${ }^{17}$ hTNFR-Is/mIgG1 chimeric receptor levels in ocular media of these treated normal rat eyes $(n=8)$ were measured by ELISA.

\section{Experimental Design and Electrotransfer to Ciliary Muscle}

Plasmid pVAX2-hTNR-Is/mIgG1 injection to the ciliary muscle and ET was performed in the treated eyes 4 days before EAU induction. Control eyes received a ciliary muscle injection of "naked plasmid" combined with ET. Thirty micrograms of plasmid (in $10 \mu \mathrm{L}$ saline) was injected in the ciliary muscle using a 30-gauge needle on a $100-\mu \mathrm{L}$ syringe (BD-microfine syringe; nm Médical, Asnière, France). An iridium/platinum electrode $(0.25-\mathrm{mm}$ diameter $)$ almost entirely covered with synthetic fluoropolymer ( $5 \mathrm{~mm}$ was left uncovered) was then introduced into the tunnel created by the needle and connected to the cathode. The platinum return anode electrode (5- $\mathrm{mm}$ long, $2.5-\mathrm{mm}$ wide, $0.3-\mathrm{mm}$ thick) was designed to fit the surface overlying the ciliary muscle and was placed facing the cathode. Optimized electric parameters consisting of eight pulses of $200 \mathrm{~V} / \mathrm{cm}, 20-\mathrm{ms}$ duration, at $5 \mathrm{~Hz}$ were delivered by an electropulsator (930 BTX; Genetronics, San Diego, CA). Another group of animals received ciliary muscle injections of the therapeutic plasmid in both eyes without ET.

Two sets of experiments were conducted until day 21 after immunization. Rats were killed by carbon dioxide inhalation. One eye of each rat was dipped in Bouin solution, embedded in paraffin, sectioned through the pupillary-optic nerve plane, and stained with hematoxylin and eosin for histology scoring, as previously described.$^{20}$ The second eye was used for immunohistochemical analysis (see Immunohistochemistry). At the end of the second experiment, three eyes from the EAU control group (ET of naked plasmid) and the ET-treated group were used to test 16 cytokines and chemokines in ocular media by multiplex bead assay (see Chemokine/Cytokine Multiplex Assay).

The third set of experiments was terminated on day 15 after immunization. Samples from control $(n=10)$ and treated $(n=10)$ groups were analyzed using the multiplex bead assay. Infiltrating inflammatory cells were collected and used for the evaluation of inducible NO synthase (NOS-2) mRNA levels (see Evaluation of NOS-2 RNA Level by RT PCR).

\section{Immunohistochemistry}

To investigate NOS-2 expression within the eye at the resolution of EAU (day 21), dual immunostaining was performed on 10- $\mu \mathrm{m}$ cryostat sections. Polyclonal rabbit anti-rat NOS-2 (1/75) (BD Transduction Laboratories, Lexington, $\mathrm{KY}$ ) and monoclonal mouse anti-rat ED1 (1/50; AbD Serotec, Hornby, ON, Canada) were used as primary antibodies. This was followed by treatment with anti-rabbit Alexa-488conjugated antibody $(1 / 250)$ and anti-mouse Texas red-conjugated antibody (1/250). Controls involved the omission of the primary antibody. Microscopic sections were viewed under the appropriate excitation filters.

\section{Evaluation of NOS-2 RNA Level by RT PCR}

Infiltrating cells from each eye were collected at day 15 after immunization by centrifugation of the ocular media, snap-frozen, and stored at $-80^{\circ} \mathrm{C}$ until use. Total RNA was isolated from cells pooled from two eyes with the same clinical score by the acid guanidinium thiocyanatephenol-chloroform method, as previously described. ${ }^{21}$ For each sample, $4 \mu \mathrm{g}$ total RNA was readjusted according to the RNA optic density at $260 \mathrm{~nm}$, transcribed, and amplified with NOS-2-specific (sense, TTT CTC TTC AAA GTC AAA TCC TAC CA; antisense, TGT GTC TGC AGA TGT GCT GAA AC) and GAPDH-specific (sense, ATG CCC CCA TGT TTG TGA TG; antisense, ATG GCA TGG ACT GTG GTC AT) primers. These primers were designed to amplify cDNA fragments of $657 \mathrm{bp}$ for NOS-2 and 162 bp for GAPDH. PCR amplification was performed according to the manufacturer's instructions ( $\sigma$-Genosys, Paris, France). PCR fragments were analyzed by $2.5 \%$ agarose gel electrophoresis and visualized by ethidium bromide staining under ultraviolet light. Relative band intensity was calculated in comparison with that for GAPDH.

\section{Chemokine/Cytokine Multiplex Assay}

Aqueous humor and vitreous collected from each eye were pooled, diluted to obtain a final volume of $25 \mu \mathrm{L}$, and subjected to multiplex bead analysis. This method uses microspheres as the solid support for immunoassays $^{22}$ and allows the titration of a greater number of cytokines with increased sensitivity than occurs with ELISA. ${ }^{23}$ 

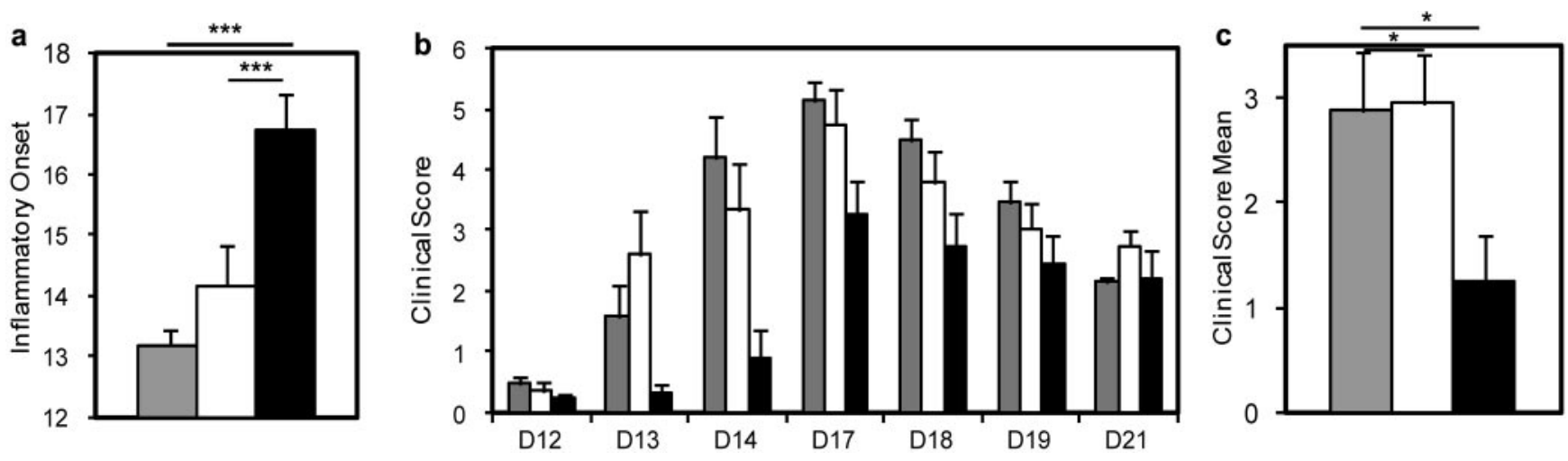

FIGURE 1. Clinical evaluation of inflammation. (a) Mean \pm SEM of first inflammation day in eyes treated with ET of naked plasmid (gray columns; pVAX2 ET; $n=10$ ), with injection of the plasmid encoding hTNFR-Is/mIgG1 (white columns; pVAX2-hTNFR-Is/mIgG1 injection; $n=14$ ) or with ET of pVAX2-hTNFR-Is/mIgG1 (black columns; $n=16$ ) in two experiments. ANOVA: $P=0.0002$; multiple comparison test of Bonferroni/Dunn: ${ }^{* * * *} P<0.005$. (b) Clinical scores by group and by day in two experiments. (c) Clinical score mean \pm SEM by group in one experiment. Clinical score mean: average of clinical scores at all time points per group. pVAX2 ET versus pVAX2-hTNFR-Is/mIgG1 ET: ${ }^{*} P=0.0474$. pVAX2-hTNFRIs/mIgG1 injection versus pVAX2-hTNFR-Is/mIgG1 ET: * $P=0.0409$.

The rat cytokine/chemokine LINCO-16plex kit (Linco Research) was purchased from Invitrogen (Cergy-Pontoise, France). Chemokines MCP-1/CCL2, MIP1 $\alpha /$ CCL3, RANTES/CCL5, IP10/CXCL10 (IFN-inducible protein-10), and GRO/KC, proinflammatory mediators IL- $1 \beta$ and TNF- $\alpha$, Th1/Th2/Th17 cytokines IL-2, IL-12p70, IFN- $\gamma /$ IL-4, IL-6, IL-10, and IL-13/IL-17, and VEGF 165 were measured according to the manufacturer's instructions. The assay was performed in a 96-well filter plate with all the assay components provided in the kit. Standard curves for each cytokine were generated with a calibration kit (Bio-Rad, Hercules, CA). All incubation steps were performed at room temperature with medium orbital agitation and in the dark to protect the beads from light. Data acquisition and analysis were performed with the manager software version 4.1 (Bioplex; Bio-Rad) with four or five logistic parameters for standard curves. Detection levels for all the cytokines were 1 to $10 \mathrm{pg} / \mathrm{mL}$.

Cytokine and chemokine levels were also measured in rat sera. Because TNF- $\alpha$ was not detectable in these samples, inguinal lymph node extracts from each rat were analyzed with multiplex bead assay.

\section{Statistical Analysis}

Results are expressed as mean \pm SEM. The Mann-Whitney $U$ test was used to determine differences between groups. $P<0.05$ was considered statistically significant.

\section{Results}

\section{Production of Therapeutic Levels of Soluble Receptors in Ocular Media by the Ciliary Muscle after Transfection of pVAX2-hTNFR-Is/mIgG1}

Eight days after $30 \mu \mathrm{g}$ plasmid ciliary muscle ET, hTNFR-Is/ mIgG1 levels of $8.7 \mathrm{ng} / \mathrm{mL} \pm 4.9 \mathrm{ng} / \mathrm{mL}$ were detected in the ocular media of treated eyes. No receptor at the sera level was found in the treated rats, demonstrating that secretion was restricted to the eye.

\section{Effect of Ciliary Muscle Electrotransfer of pVAX2- hTNFR-Is/mIgG1 on Clinical Signs of EAU}

Four days before immunization by S-Ag for EAU induction, eyes treated by ET of the plasmid encoding hTNFR-Is/mIgG1 showed significantly delayed onset of clinical disease and markedly reduced severity of EAU compared with control eyes treated with naked plasmid (pVAX2 ET). The timing of detectable inflammation in the treated eyes was $16.8 \pm 1.1$ days compared with $13.2 \pm 0.4$ in control eyes $(P=0.035)$. Injec- tion of the therapeutic plasmid (pVAX2-hTNFR-Is/mIgG1) in the ciliary muscle, without ET, did not influence the timing of the initial signs of inflammation (Fig. 1a). Inflammatory cell infiltration was lower in treated eyes at day 14 than in control eyes, suggesting that treatment impeded the early infiltration of ocular tissue by inflammatory cells. Later the inflammation was similar in treated eyes and control eyes (Fig. 1b). When considering the means of all inflammation scores at all time points, clinical scores of EAU were lower in the group of eyes treated with pVAX2-hTNFR-Is/mIgG1 ET (1.25 \pm 0.043$)$ than in the control group receiving naked plasmid ET $(2.88 \pm 0.57 ; P=$ $0.0474)$. Moreover, the group of eyes receiving only ciliary muscle injection of the therapeutic plasmid, without ET, demonstrated no decrease in clinical score intensity $(2.95 \pm 0.46$; Fig. 1c).

\section{Photoreceptor Rescue}

EAU causes severe damage to the retina, including total destruction of the outer nuclear layer, subretinal exudates, hemorrhage, RPE lesions, and inflammation of the choroid (Fig. 2). These EAU-associated manifestations reached their peak 21 days after immunization, when the severity of histologic retinal damage was significantly decreased in eyes treated with ET of hTNFR-Is/mIgG1 plasmid $(1.4 \pm 0.37$ compared with $4.0 \pm$ 0.32 in the control untreated eyes; $P=0.0039$; Fig. 2a). Subsequently, retinal thickness (Fig. 2b) and number of photoreceptor nuclei (Fig. 2c) were significantly higher in treated eyes, demonstrating that significant rescue of photoreceptors was achieved.

\section{Reduction of NOS-2 Expression in Infiltrating Cells}

We observed that despite the marked clinical beneficial effects observed in the rat eyes treated by injection of pVAX2-hTNFRIs/mIgG1 combined with ET, the intraocular infiltration of monocytes and macrophages was not abolished (Fig. 3a). In control eyes, numerous ED1-positive macrophages colocalized with NOS-2 in the anterior chamber, whereas in the vitreous and the retina, few cells expressing NOS- 2 could be detected in the pVAX2-hTNFR-Is/mIgG1 ET-treated eyes. Furthermore, in the control eye retina, some of microglial cells expressed NOS-2, but in treated eyes, ED1-positive microglial cells were mainly NOS- 2 negative.

To ascertain the effect of treatment on NOS-2 expression, infiltrating cells were collected from the ocular media at the 


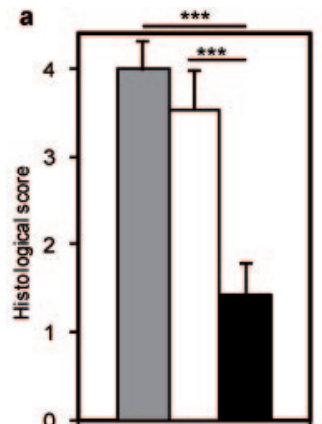

d

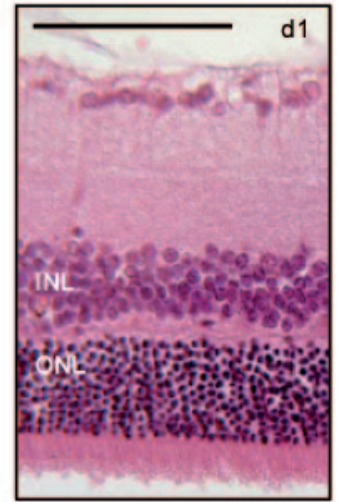

b
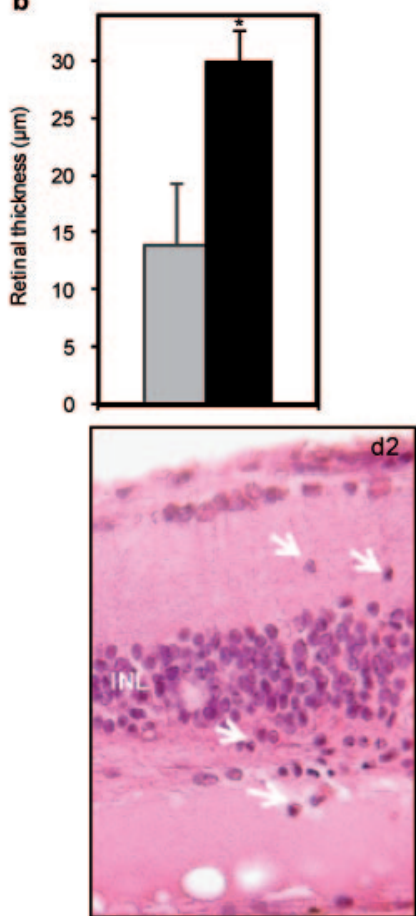

onset of disease, and NOS-2 expression was analyzed by RTPCR in control and treated eyes (Fig. 3b). This experiment confirmed that NOS-2 expression was reduced in infiltrating cells collected from treated eyes, suggesting that TNF- $\alpha$ blockade also induced a change in macrophage phenotype expression.

\section{Shift toward a Th2 Cytokine Profile in Ocular Media}

To determine the activation state of inflammatory cells in ocular media, cytokine and chemokine profiles of eyes treated
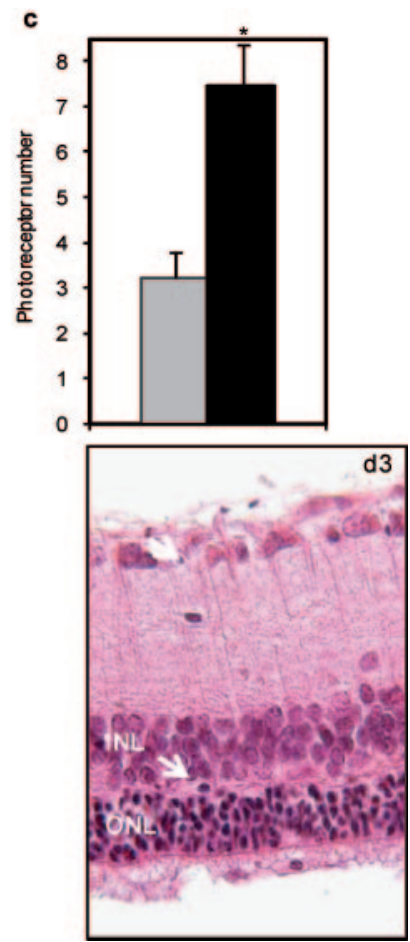

Figure 2. Histologic scores at day 21 after immunization. (a) Mean \pm SEM of histologic scores in eyes treated with ET of naked plasmid (gray columns; pVAX2 ET; $n=5$ ), with injection of pVAX2-hTNFR-Is/ mIgG1 (white columns; $n=7$ ) or with ET of pVAX2-hTNFR-Is/mIgG1 (black columns, $n=8$ ). ANOVA: $P=0.012$; multiple comparison test of Bonferroni/Dunn: ${ }^{* * *} P<0.005$. (b) Thickness of photoreceptor layer and (c) number of photoreceptor nucleus mean \pm SEM in eyes treated with naked plasmid ET (gray columns) or ET of the therapeutic plasmid (black columns). Thickness comparison: ${ }^{*} P=0.023$; photoreceptor number comparison: ${ }^{*} P=$ 0.0147. (d) Retinal sections. (d1) Normal rat retina. Scale bar, $100 \mu \mathrm{m}$. INL, inner nuclear layer; ONL, outer nuclear layer. (d2) Control eye with EAU (pVAX2 ET). More inflammatory cells (arrowhead) and subretinal exudates are observed in control eyes than in pVAX2-hTNR-Is/mIgG ETtreated eye. (d3) pVAX2-hTNFR-Is/ mIgG1 ET-treated eye with EAU.

with the plasmid encoding hTNFR-Is/mIgG1 were compared with those of naked plasmid ET-treated eyes on day 15. At this time, the mean clinical score of EAU was significantly decreased in the treated group $(1.6 \pm 0.34)$ compared with the control group $(3.4 \pm 0.68 ; P=0.0359)$.

Ocular Media Levels of Cytokines/Chemokines at the Onset of Inflammation. All tested cytokines and chemokines except MIP-1 $\alpha$, which was below the detection level for both groups, were found in ocular media (Fig. 4). TNF- $\alpha$ was significantly reduced in the ocular media of treated eyes compared with those of control eyes (Fig. 4a).
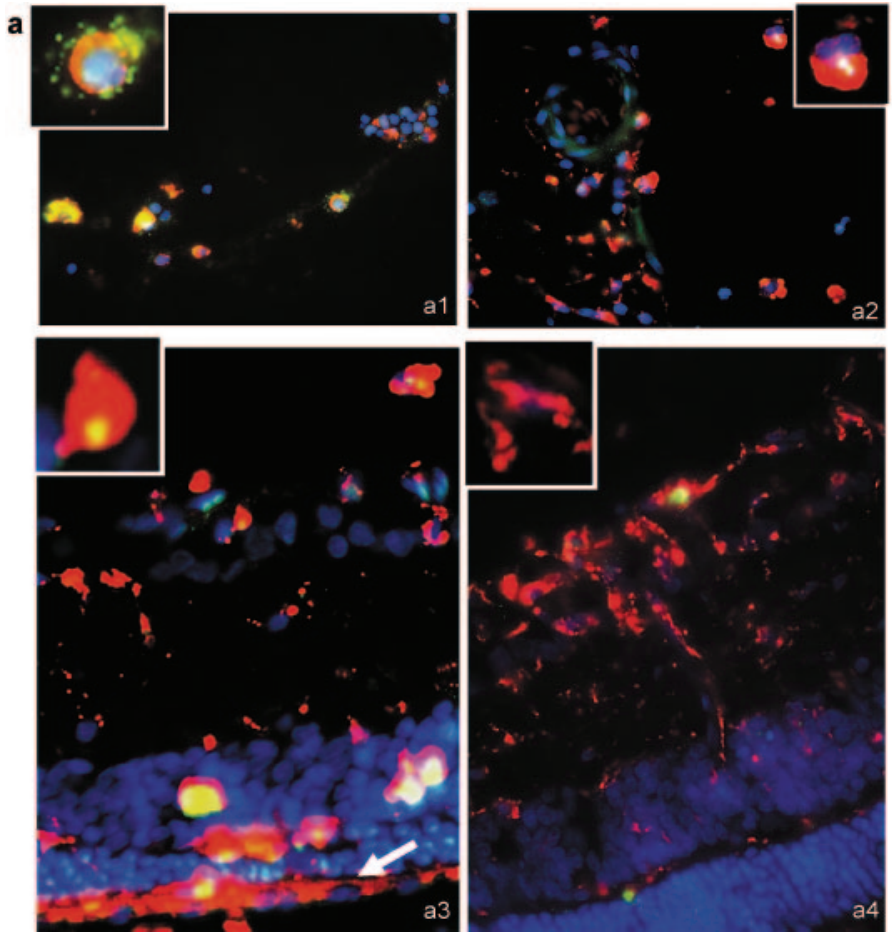

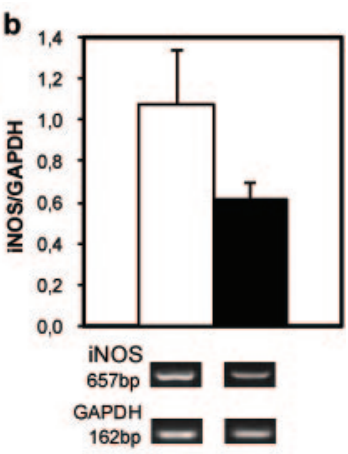

Figure 3. Effect of TNF- $\alpha$ blockade on NOS-2 expression by infiltrating and resident cells. (a) Immunohistochemical labeling of NOS-2 expressed by infiltrating macrophage (a1, a2) and by resident cells $(\mathbf{a} 3, \mathbf{a} 4)$ at day 21 after immunization. Dual immunofluorescence staining shows the colocalization of NOS-2-A488 (green) and ED1-Texas red in EAU control eye (a1, a3) and in pVAX2-hTNR-Is/ mIgG1 ET-treated eye (a2, a4). (a3) Arrowhead highlights the large number of activated macrophages in the subretinal space with the destruction of the photoreceptor cells. (b) Effect on NOS-2 mRNA expression by infiltrating ocular cells at day 15 after immunization. Mean \pm SEM relative band intensity determined after the detection of NOS-2 and GAPDH mRNA expression in eyes treated with naked plasmid ET (white columns; $n=$ 5) or with pVAX2-hTNR-Is/mIgG1 ET (black columns; $n=5$ ). Representative RT-PCR products visualized by ethidium bromide staining. 

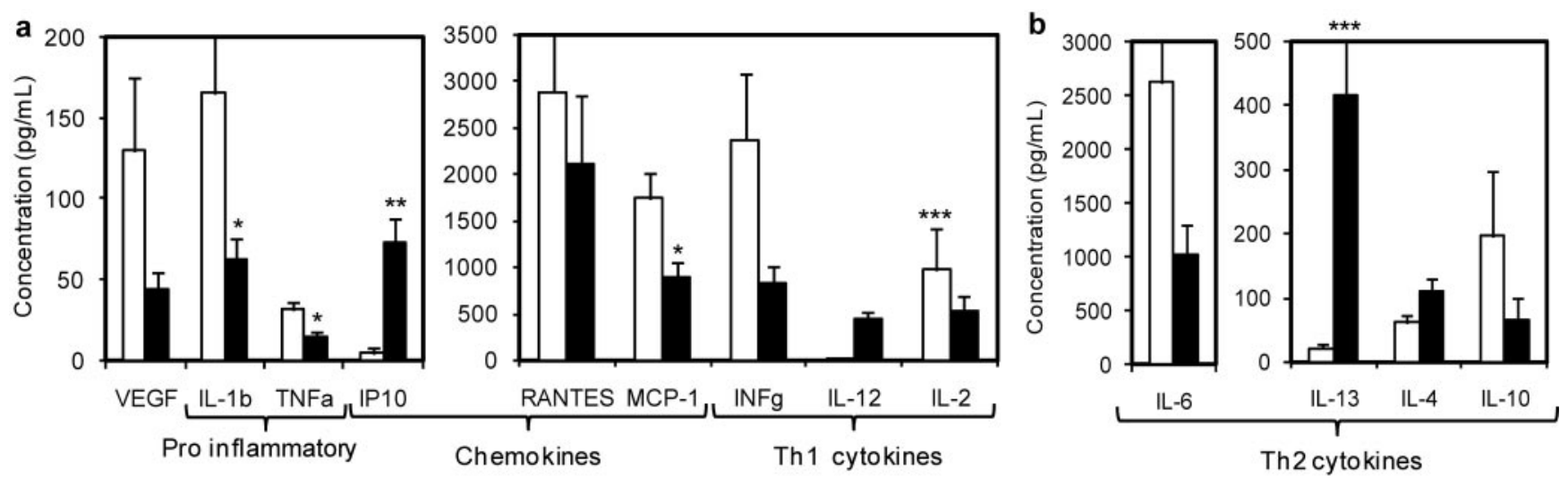

\section{c $P$ value of the non parametric Wilcoxon-Mannwhitney test between control and treated eyes}

\begin{tabular}{lllllllllllll} 
VEGF & IL-1b & TNFa & IP10 & Rantes & MCP1 & IFNg & IL-12p70 & IL-2 & IL-6 & IL-13 & IL-4 & IL-10 \\
\hline NS & 0.036 & 0.0173 & 0.0018 & NS & 0.0173 & NS & 0.0002 & $(0.08)$ & NS & 0.0002 & 0.0539 & NS
\end{tabular}

FIGURE 4. Cytokine and chemokine profiles in ocular media 15 days after immunization. (a) VEGF, chemokine, proinflammatory, Th1 cytokine, and (b) Th2 cytokine levels in pVAX2-hTNR-Is/mIgG1 ET-treated eyes (black columns; $n=10$ ) compared with those of naked plasmid ET-treated eyes (white columns; $n=10$ ). ${ }^{*} P<0.05 ;{ }^{* *} P<0.01 ;{ }^{* * *} P<0.001$. (c) Comparison of cytokine and chemokine levels in ocular media.

In the inguinal lymph nodes, however, TNF- $\alpha$ was similar in the control group treated with the naked plasmid (pVAX2 ET; $62 \pm 2 \mathrm{pg} / \mathrm{mL}$ ) and in the group treated with the plasmid encoding hTNFR-Is/mIgG1 (69 $\pm 9 \mathrm{pg} / \mathrm{mL} ; P>0.05)$. These observations demonstrate that appropriate plasmid ET to the ciliary muscle has a marked influence on the local intraocular production of TNF- $\alpha$ without any significant influence on its systemic production.

In treated eyes, TNF- $\alpha$ blockade significantly lowered the levels of the proinflammatory cytokine IL- $1 \beta$ and of the chemokine MCP-1 (Fig. 4a), significantly increased the levels of the anti-inflammatory cytokine IL-13, and tended to increase the level of IL-4 ( $P=0.0539$; Fig. 4b). Surprisingly, the active p70 subunit of IL-12 and IP-10 levels was significantly increased in eyes treated with TNF- $\alpha$ soluble receptor (Fig. 4a). As shown by correlations between pairs of cytokines in the ocular media, TNF- $\alpha$ levels appeared directly correlated with MCP-1 levels and inversely correlated with those of IL-12p70, IL-13, IL-4, and IP-10 (Fig. 5). Interestingly, VEGF and IFN- $\gamma$ levels were drastically decreased in treated eyes, but the decrease did not reach statistical significance. However, GRO/KC $(821 \pm 178$ pg/mL in the control group vs. $1198 \pm 178 \mathrm{pg} / \mathrm{mL}$ in the treated group) and IL-17 (1562 $\pm 980 \mathrm{pg} / \mathrm{mL}$ in the control group vs. $1456 \pm 359 \mathrm{pg} / \mathrm{mL}$ in the treated group) were not influenced by TNF- $\alpha$ blockade

Effect of TNF- $\alpha$ Blockade on the Onset of EAU and Its Relation with Cytokine/Chemokine Production in the Ocular Media. As illustrated in Figure 6, anti-TNF- $\alpha$ treatment generated a deviation toward anti-inflammatory cell regulatory mechanisms. This phenomenon occurred as soon as the early stages of initial clinical disease were detected.

Ocular Media Levels of Cytokines/Chemokines at the Resolution of Disease. Cytokine and chemokine responses observed at day 21 after immunization confirmed that the treatment modified immune response (Fig. 7). At the resolution of the disease (day 21), TNF- $\alpha$ blockade was associated with a marked increase of Th2 cytokine (IL-13 and IL- 4 but also IL-6) levels in ocular media. As observed at day 15, MCP-1 and VEGF levels were drastically reduced by treatment. Moreover, IL12p70 and IP10 concentrations were strongly increased and were associated with high levels of IFN- $\gamma$.
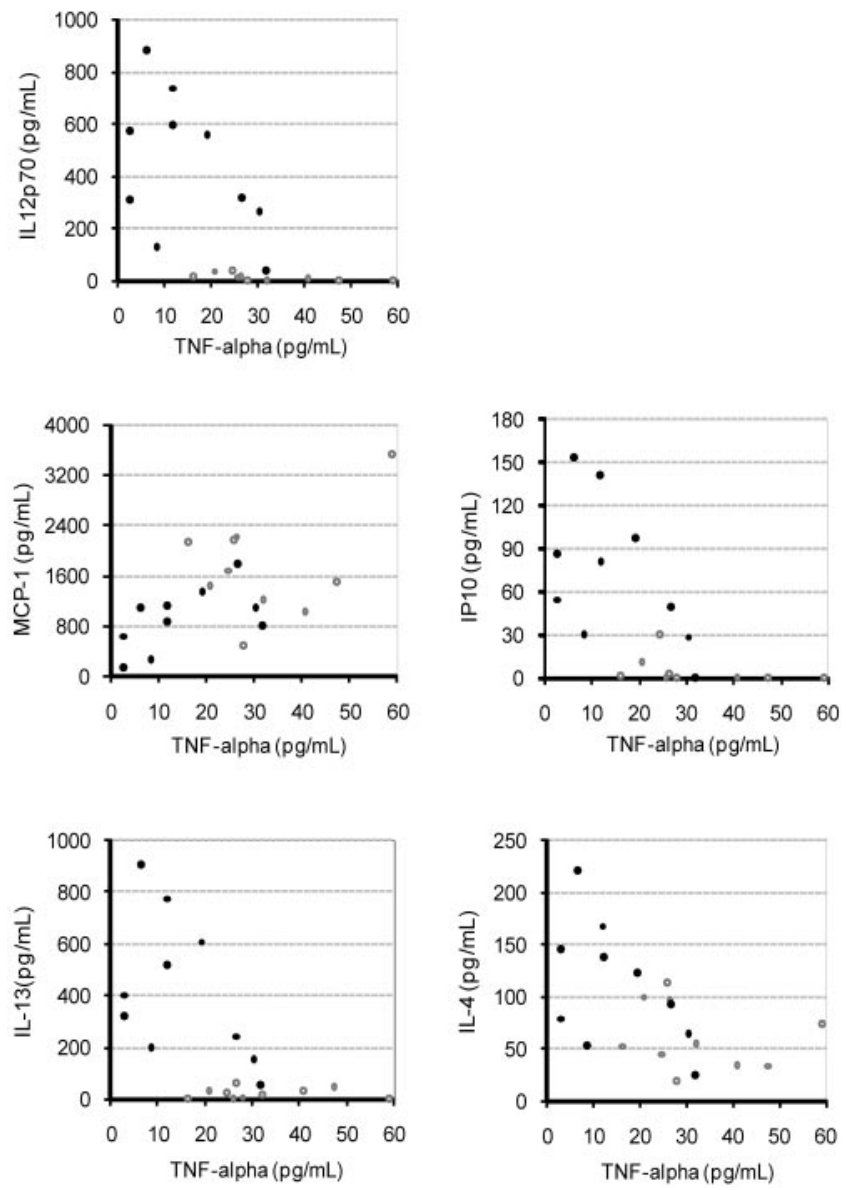

FIGURE 5. Cytokines and chemokines significantly altered by TNF- $\alpha$ blockade in EAU control eyes $(\bigcirc$ pVAX2 ET) and in eyes treated with the plasmid encoding hTNR-Is/mIgG1 (O) at day15 after immunization. Data represent the association between TNF- $\alpha$ and cytokines/chemokines in which significant correlations were observed. For each association, the Pearson correlation $(r)$ and significance $(P)$ for all points are indicated. 

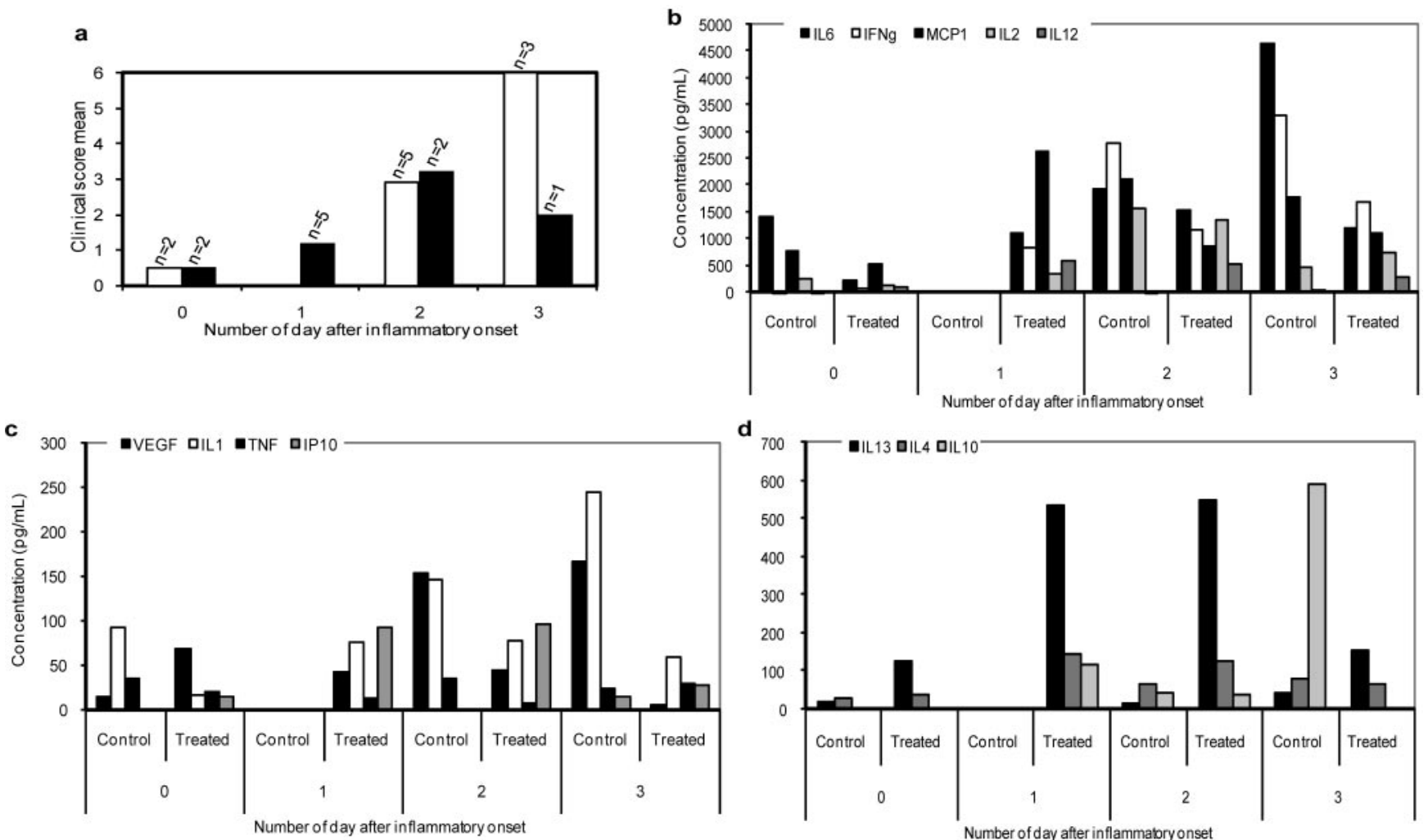

FigURE 6. Representation of cytokine/chemokine levels, depending on the number of days after inflammation onset. (a) Clinical score means in eyes treated with naked plasmid ET (white columns; $n=10$ ) or ET of the plasmid encoding hTNFR-Is/mIgG1 (black columns; $n=10$ ). For each day after inflammation onset, the number of eyes by group is indicated at the top of the corresponding bar. (b-d) Ocular media cytokine and chemokine levels by group (Control, pVAX2 ET-treated eyes; Treated, pVAX2-hTNR-Is/mIgG1 ET-treated eyes) and by number of days after EAU onset.

At the time of resolution, RANTES expression in the control group was strongly increased compared with the level observed at the onset of inflammation. In contrast, at day 21 , eyes treated with TNF- $\alpha$ soluble receptor showed RANTES levels lower than at day 15. A decrease was observed in IL-17 levels in both groups compared with the concentrations measured at day15, but this decrease was more important in treated eyes.

\section{Discussion}

Uveitis remains one of the leading causes of blindness worldwide. Loss of vision can be delayed or prevented by appropri- ate treatment. Given that clinical uveitis is a heterogeneous group of diseases, experimental models such as EAU in the rat have provided useful insight regarding potential immune mechanisms and new therapeutic avenues. These methods include intravitreous injection of immunosuppressant agents such as tacrolimus (FK506), ${ }^{24}$ a monoclonal antibody directed at CD200R (DX109), ${ }^{25}$ and regulatory T cells. ${ }^{20}$ When considering such treatments for patients with uveitis, these intraocular injections should be repeated as long as the inflammatory process is taking place. Viral gene therapy has been used to allow for sustained delivery of immunomodulatory molecules within the eye. ${ }^{26-30}$ In particular, adenoassociated viruses have a

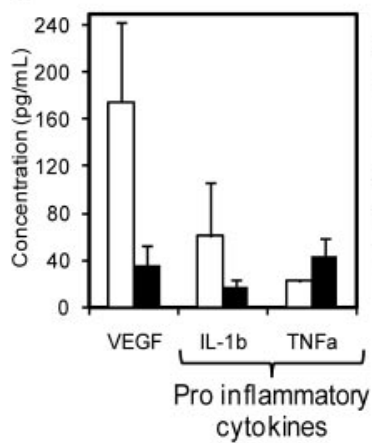

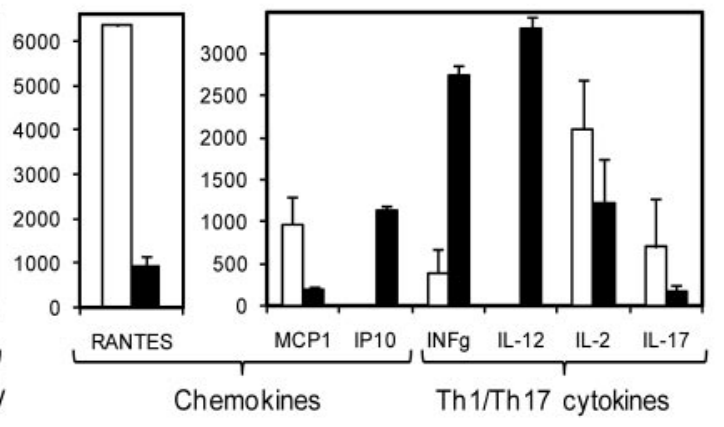

b

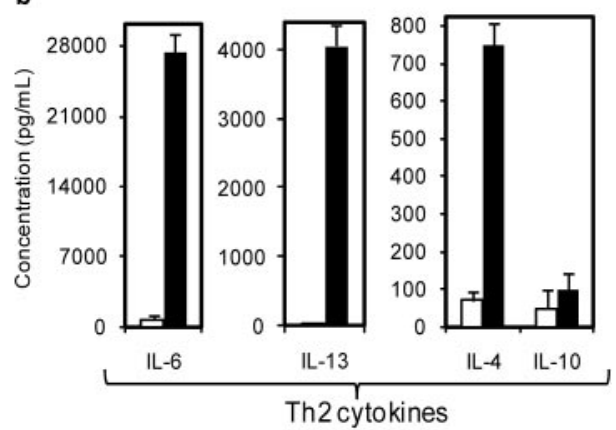

FIGURE 7. Cytokine and chemokine profiles in ocular media 21 days after immunization. (a) VEGF, chemokine, proinflammatory, Th1/Th17 cytokine and (b) Th2 Cytokine levels in pVAX2(hTNFR-Is/mIgG1) ET-treated eyes (black columns; $n=3$ ) compared with naked plasmid ET-treated eyes (white columns; $n=3$ ). 
been shown to transfect efficiently a variety of retinal cell types, and they are undergoing testing in clinical trials for gene replacement in inherited retinal degeneration. ${ }^{31}$ Nonviral vector strategies offer the advantages of increased safety and reduced risks for unwarranted immune reactions.

We have developed a nonviral gene therapy method with a well-controlled ET strategy. Indeed, ET leads to a high transfection rate compared with other nonviral techniques, such as liposomes ${ }^{32}$ and cationic lipids, ${ }^{33}$ notably in promoting DNA migration to the nucleus. ${ }^{34}$ We refined this technique and used the intraocular ciliary muscle as the site for gene transfection and expression. We showed that long-lasting intraocular expression of therapeutic proteins can be achieved ${ }^{17}$ and then observed that the production of proteins in the ocular media could last for at least 9 months (Touchard E, unpublished observations, 2007).

Many reports have displayed the importance of TNF- $\alpha$ in EAU and uveitis. ${ }^{10,16}$ Clinical onset of disease and histologic damage are suppressed when TNF- $\alpha$ activity is neutralized during the afferent phase of the immune response with systemic administration of TNF- $\alpha$ inhibitors. ${ }^{7,12,35,36}$ Based on these data and on the fact that we have previously shown that our gene delivery method induces a peak of expression 8 days after ET ${ }^{17}$ the appropriate protocol was to treat rat eyes 4 days before immunization. The present study shows that the local intraocular production of soluble p55 receptors in the ocular media had beneficial effects on the time course and the severity of EAU. Although cell trafficking was not impaired, neutralizing TNF- $\alpha$ activity suppressed effector mechanisms of the proinflammatory cells and reduced apoptosis in the retina. These results confirmed that TNF blockade is effective during the early phase of the immune response. Consequently, because a delay is needed before the p 55 TNF- $\alpha$ receptor fusion protein is produced in the eye, ET of plasmid encoding the therapeutic protein would be more appropriate for the treatment of patients with chronic uveitis and recurrences.

Multiplex immunoassay is a powerful method and demonstrates that the effect on photoreceptor rescue is attributed not only to chimeric TNF- $\alpha$ receptor secretion but also to a sustained change in cytokine and chemokine expression.

On one hand, neutralization of the intraocular TNF- $\alpha$ induces a local modulation of the immune response as early as the onset of the disease (day 15) through a reduction of the chemokine MCP-1 and the proinflammatory cytokines (IL-1- $\beta$, TNF- $\alpha$ ) together with low NOS-2 expression. These changes are observed with a switch toward anti-inflammatory cytokines, primarily IL-13 and IL-4. Injection of IL-13 into the anterior chamber has already been reported to inhibit innate response in EIU by reducing cytokine (TNF- $\alpha$, IL-1 $\beta$, IL- 6 ) and chemokine (MCP-1 and MIP-2) mRNA expression, whereas IFN- $\gamma$ was upregulated in the iris-ciliary body. ${ }^{37}$ Altogether, analysis of the activity of infiltrating cells suggests that the anti-TNF- $\alpha$ treatment led to an anti-inflammatory environment because of a change of macrophage phenotype in treated animals. Such a change in macrophage phenotype, signed by the reduced expression of inflammatory mediators, has already been described after IL-13 treatment. ${ }^{38}$

On the other hand, TNF- $\alpha$ blockade was correlated with the IP-10 and IL-12 p70 levels. IP10 is a chemokine known to recruit $\mathrm{CD}^{+}{ }^{\mathrm{T}}$ cells, ${ }^{39}$ and IL-12p70 is a cytokine that plays a critical role in Th1 differentiation but has ambiguous inflammatory effects. ${ }^{40,41}$ In addition to $\mathrm{CD}^{+}$Th 1 cells, the recently discovered IL-17-producing cells, Th17 cells, are pathogenic effectors in EAU. ${ }^{40,42}$ Whereas at day 15 there was no difference in intraocular IL-17 levels between treated and controls, at day 21 a decrease of IL-17 was observed in both groups, but this decrease was stronger in treated eyes. Concerning the effect of the treatment on chemokine levels, the inhibitory effect on MCP1 and the trend to a decrease of RANTES levels at day 15 was amplified even at the time of resolution. At day 21, a simultaneous dramatic increase of Th2 cytokines, IL-6, IL-13, and IL-4, and a trend to an increase of IL-10 were also observed.

Thus, cytokine/chemokine profiles revealed that the treatment of this autoimmune inflammation model with p 55 TNF- $\alpha$ soluble receptors blocked the innate response by reducing the production of proinflammatory mediators despite few effects on adaptive response to EAU. Therefore, additional immunosuppressive agents may be required to neutralize these mediators in this type of inflammatory processes.

In patients, the $\mathrm{p} 55$ receptor fusion protein was efficient in severe noninfectious posterior segment inflammations. Repeated intravenous infusions, associated with numerous secondary complications and risks, are required to maintain the therapeutic benefit. ${ }^{43}$ In our experimental studies, no circulating soluble receptors were measured within the sera of treated rats. In addition, TNF- $\alpha$ production by the inguinal lymph nodes of treated animals was not affected, suggesting that no systemic adverse effects observed after systemic injection of TNF- $\alpha$ inhibitors should result from this newly devised intraocular immunomodulation. Our results clearly show that local production of TNF- $\alpha$ receptor was effective at reducing EAU through the local reduction of proinflammatory mediators and the shift to anti-inflammatory mediators. This local modulation of EAU, a systemic disease, was sufficient to reduce ocular inflammation. Consequently, the sustained intraocular production of p55 soluble receptors with ciliary muscle ET may offer an efficient therapeutic option for the treatment of uveitis refractory to conventional immunosuppressive therapy.

Interestingly, we found that the secretion of anti-TNF- $\alpha$ chimeric protein is also accompanied by a marked decrease of VEGF levels in ocular media. This incidental finding may also have some therapeutic implications associated with the decrease of macular edema often occurring in patients with chronic uveitis.

In conclusion, we have demonstrated that with the use of a nonviral gene therapy strategy, intraocular production of soluble $\mathrm{p} 55$ receptors to TNF- $\alpha$ reduces proinflammatory cytokine/ chemokine levels and increases protective cytokine release. Along with the induced cytokine repertoire changes, markedly reduced severity of clinical disease and prevention of severe retinal damage are observed. Therefore, this study highlights the potential therapeutic benefits that can be achieved by self-therapy when using the ocular ciliary muscle as a local "factory" for the production of appropriate therapeutic molecules. It is our hope that this therapeutic strategy can be implemented and adapted for additional ocular diseases. Various potential diseases are now under investigation in our laboratory.

\section{References}

1. Forrester JV. Intermediate and posterior uveitis. Chem Immunol Allergy. 2007;92:228-243.

2. Yeh S, Faia LJ, Nussenblatt RB. Advances in the diagnosis and immunotherapy for ocular inflammatory disease. Semin Immunopatbol. 2008;30:145-164.

3. Curnow SJ, Falciani F, Durrani OM, et al. Multiplex bead immunoassay analysis of aqueous humor reveals distinct cytokine profiles in uveitis. Invest Ophthalmol Vis Sci. 2005;46:4251-4259.

4. Takase H, Futagami Y, Yoshida T, et al. Cytokine profile in aqueous humor and sera of patients with infectious or noninfectious uveitis. Invest Opbthalmol Vis Sc. 2006;47:1557-1561.

5. Sijssens KM, Rijkers GT, Rothova A, Stilma JS, Schellekens PA, de Boer JH. Cytokines, chemokines and soluble adhesion molecules in aqueous humor of children with uveitis. Exp Eye Res. 2007;85: 443-449. 
6. Ahn JK, Yu HG, Chung H, Park YG. Intraocular cytokine environment in active Behçet uveitis. Am J Ophthalmol. 2006;142:429434.

7. Dick $\mathrm{AD}$, McMenamin $\mathrm{PG}$, Körner $\mathrm{H}$, et al. Inhibition of tumor necrosis factor activity minimizes target organ damage in experimental autoimmune uveoretinitis despite quantitatively normal activated T cell traffic to the retina. Eur J Immunol. 1996;26: $1018-1025$.

8. Maini RN, Taylor PC. Anti-cytokine therapy for rheumatoid arthritis. Annu Rev Med. 2000;51:207-229.

9. Papadakis KA, Targan SR. Role of cytokines in the pathogenesis of inflammatory bowel disease. Annu Rev Med. 2000;51:289-298.

10. Dick AD, Forrester JV, Liversidge J, Cope AP. The role of tumour necrosis factor (TNF-alpha) in experimental autoimmune uveoretinitis (EAU). Prog Retin Eye Res. 2004;23:617-637.

11. Hankey DJ, Lightman SL, Baker D. Interphotoreceptor retinoid binding protein peptide-induced uveitis in B10.RIII mice: characterization of disease parameters and immunomodulation. Exp Eye Res. 2001;72:341-350.

12. Robertson M, Liversidge J, Forrester JV, Dick AD. Neutralizing tumor necrosis factor-alpha activity suppresses activation of infiltrating macrophages in experimental autoimmune uveoretinitis. Invest Ophthalmol Vis Sci. 2003;44:3034-3041.

13. Lin J, Ziring D, Desai S, et al. TNF-alpha blockade in human diseases: an overview of efficacy and safety. Clin Immunol. 2008; 126:13-30.

14. Kempen JH, Gangaputra S, Daniel E, et al. Long-term risk of malignancy among patients treated with immunosuppressive agents for ocular inflammation: a critical assessment of the evidence. Am J Ophthalmol. 2008;146:802-812

15. Nussenblatt R. Treating intraocular inflammatory disease in the 21st century. Arch Ophthalmol. 2005;123:1000-1001.

16. Hale $S$, Lightman $S$. Anti-TNF therapies in the management of acute and chronic uveitis. Cytokine. 2006;33:231-237.

17. Bloquel C, Bejjani R, Bigey P, et al. Plasmid ET of eye ciliary muscle: principles and therapeutic efficacy using hTNF-alpha soluble receptor in uveitis. FASEB J. 2006;20:389-91.

18. De Kozak Y, Sakai J, Thillaye B, Faure JP. S-antigen-induced experimental autoimmune uveoretinitis in rats. Curr Eye Res. 1981;1: 327-337.

19. Bloquel C, Bessis N, Boissier MC, Scherman D, Bigey P. Gene therapy of collagen-induced arthritis by ET of human tumor necrosis factor-alpha soluble receptor I variants. Hum Gene Ther. 2004;15:189-201.

20. Terrada C, Fisson S, De Kozak Y, et al. Regulatory T cells control uveoretinitis induced by pathogenic Th1 cells reacting to a specific retinal neoantigen. J Immunol. 2006;176:7171-7179.

21. Thillaye-Goldenberg B, Goureau O, Naud M-C, de Kozak Y. Delayed onset and decreased severity of experimental autoimmune uveoretinitis in mice lacking nitric oxide synthase type 2. J Neuroimmunol. 2000;110:31-44.

22. Vignali DA. Multiplexed particle-based flow cytometric assays J Immunol Methods. 2000;243:243-255.

23. Ooi KG, Galatowicz G, Towler HM, Lightman SL, Calder VL. Multiplex cytokine detection versus ELISA for aqueous humor: IL-5, IL-10, and IFN $\gamma$ profiles in uveitis. Invest Ophthalmol Vis Sci. 2006; $47: 272-277$.

24. Oh-i K, Keino H, Goto $\mathrm{H}$, et al. Intravitreal injection of tacrolimus (FK506) suppresses ongoing experimental autoimmune uveoretinitis in rats. Br J Ophthalmol. 2007;91:237-242.

25. Copland DA, Calder CJ, Raveney BJ, et al. Monoclonal antibodymediated CD200 receptor signaling suppresses macrophage acti- vation and tissue damage in experimental autoimmune uveoretinitis. Am J Pathol. 2007;171:580-588.

26. De Kozak Y, Andrieux K, Villarroya H, et al. Intraocular injection of tamoxifen-loaded nanoparticles: a new treatment of experimental autoimmune uveoretinitis. Eur J Immunol. 2004;34:37023712.

27. Smith JR, Verwaerde C, Rolling F, et al. Tetracycline-inducible viral interleukin-10 intraocular gene transfer, using adeno-associated virus in experimental autoimmune uveoretinitis. Hum Gene Ther. 2005;16:1037-1046

28. Broderick CA, Smith AJ, Balaggan KS, et al. Local administration of an adeno-associated viral vector expressing IL-10 reduces monocyte infiltration and subsequent photoreceptor damage during experimental autoimmune uveitis. Mol Ther. 2005;12:369-373.

29. Verwaerde C, Naud MC, Delanoye A, et al. Ocular transfer of retinal glial cells transduced ex vivo with adenovirus expressing viral IL-10 or CTLA4-Ig inhibits experimental autoimmune uveoretinitis. Gene Ther. 2003;10:1970-1981.

30. De Kozak Y, Thillaye-Goldenberg B, Naud MC, Da Costa AV, Auriault C, Verwaerde C. Inhibition of experimental autoimmune uveoretinitis by systemic and subconjunctival adenovirus-mediated transfer of the viral IL-10 gene. Clin Exp Immunol. 2002;130: 212-223.

31. Buch PK, Bainbridge JW, Ali RR. AAV-mediated gene therapy for retinal disorders: from mouse to man. Gene Ther. 2008;15:849857.

32. Pleyer U, Dannowski H. Delivery of genes via liposomes to corneal endothelial cells. Drug News Perspect. 2002;15:283-289.

33. Pitkänen L, Ruponen M, Nieminen J, Urtti A. Vitreous is a barrier in nonviral gene transfer by cationic lipids and polymers. Pharm Res. 2003;20:576-583.

34. Rols MP. Mechanism by which electroporation mediates DNA migration and entry into cells and targeted tissues. Methods Mol Biol. 2008;423:19-33.

35. Sartani G, Silver PB, Rizzo LV, et al. Anti-tumor necrosis factor alpha therapy suppresses the induction of experimental autoimmune uveoretinitis in mice by inhibiting antigen priming. Invest Ophthalmol Vis Sci. 1996;37:2211-2218.

36. Dick AD, Duncan L, Hale G, Waldmann H, Isaacs J. Neutralizing TNF-alpha activity modulates T-cell phenotype and function in experimental autoimmune uveoretinitis. J Autoimmun. 1998;11: 255-264.

37. Lemaitre C, Thillaye-Goldenberg B, Naud MC, de Kozak Y. The effects of intraocular injection of interleukin-13 on endotoxininduced uveitis in rats. Invest Ophthalmol Vis Sci. 2001;42:20222030.

38. de Kozak Y, Omri B, Smith JR, Naud MC, Thillaye-Goldenberg B, Crisanti P. Protein kinase Czeta (PKCzeta) regulates ocular inflammation and apoptosis in endotoxin-induced uveitis (EIU): signaling molecules involved in EIU resolution by PKCzeta inhibitor and interleukin-13. Am J Pathol. 2007;170:1241-1257.

39. Amescua G, Collings F, Sidani A, et al. Effect of CXCL-1/KC production in high risk vascularized corneal allografts on $\mathrm{T}$ cell recruitment and graft rejection. Transplantation. 2008;85:615-625.

40. Luger D, Caspi RR. New perspectives on effector mechanisms in uveitis. Semin Immunopathol. 2008;30:135-143.

41. De Kozak Y, Verwaerde C. Cytokines in immunotherapy of experimental uveitis. Int Rev Immunol. 2002;21:231-253.

42. Amadi-Obi A, Yu CR, Liu X, et al. TH17 cells contribute to uveitis and scleritis and are expanded by IL-2 and inhibited by IL-27/ STAT1. Nat Med. 2007;13:711-718.

43. Murphy CC, Greiner K, Plskova J, et al. Neutralizing tumor necrosis factor activity leads to remission in patients with refractory noninfectious posterior uveitis. Arch Ophthalmol. 2004;122:845-851. 